\title{
Four Flaps Technique for Neoumbilicoplasty
}

\author{
Young Taek Lee, Chan Kwon, Seung Chul Rhee, Sang Hun Cho, Su Rak Eo \\ Department of Plastic and Reconstructive Surgery, Dongguk University Ilsan Hospital, Dongguk University College of Medicine, Goyang, \\ Korea
}

The absence or disfigurement of the umbilicus is both cosmetically and psychologically distressing to patients. The goal of aesthetically pleasing umbilical reconstruction is to create a neoumbilicus with sufficient depth and good morphology, with natural-looking superior hooding and minimal scarring. Although many reports have presented techniques for creating new and attractive umbilici, we developed a technique that we term the "four flaps technique" for creating a neoumbilicus in circumstances such as the congenital absence of the umbilicus or the lack of remaining umbilical tissue following the excision of a hypertrophic or scarred umbilicus. This method uses the neighboring tissue by simply elevating four flaps and can yield sufficient depth and an aesthetically pleasing shape with appropriate superior hooding.

Keywords Umbilicus / Abdominoplasty / Abdominal wall
Correspondence: Su Rak Eo Department of Plastic and Reconstructive Surgery, Dongguk University Ilsan Hospital, Dongguk University College of Medicine, 27 Dongguk-ro, Ilsandong-gu, Goyang 410-773, Korea Tel: +82-31-961-7342 Fax: +82-31-961-7347 E-mail: surakeo@yahoo.com

No potential conflict of interest relevant to this article was reported.

Received: 30 Oct 2014 - Revised: 5 Jan $2015 \bullet$ Accepted: 19 Jan 2015

pISSN: 2234-6163・ elSSN: 2234-6171・http://dx.doi.org/10.5999/aps.2015.42.3.351・ Arch Plast Surg 2015;42:351-355

\section{INTRODUCTION}

As a major aesthetic component of the abdominal wall, the umbilicus is the only scar tissue in the human body which normally remains after birth. An aesthetically pleasing umbilicus has sufficient dimpling and natural superior hooding with an almond shape. Umbilicoplasty is required not only in conditions such as congenital absence due to bladder exstrophy, gastroschisis, omphalocele, and cloacal exstrophy, but also in cases of a protruding umbilicus, traumatic umbilical malformation, and after abdominoplasty, breast reconstruction using abdominal tissues, umbilical herniorrhaphy, and laparotomy. Various methods have been employed in order to reconstruct the umbilicus, including the purse-string method [1], the use of several local flaps [2-6], and the use of an ear conchal cartilage graft [7]. Every method has advantages and disadvantages; however, none of the above methods can guarantee optimal results. Here, we report our experience with umbilical reconstruction using a technique we have termed the "four flaps technique" (Fig. 1), which we suggest may be an easy, simple, safe, and reliable technique, especially in cases where no remaining umbilical tissue is present.

\section{IDEA}

In our new umbilicoplasty technique, we only utilize neighboring abdominal soft tissue instead of using remaining umbilical tissue, which we regard as the most distinctive and creative feature of our technique. Three of the four newly created flaps (those in the 3, 6, and 9 oclock directions) are elevated and firmly anchored to the anterior rectus fascial layer. This particular surgical procedure has not previously been reported. Our technique was designed for individuals who congenitally lack umbilical tissues (Fig. 2) or for use after the excision of a severely hypertrophic and disfigured umbilicus (Fig. 3). In both cases, no umbilical tissue was available for use, leaving only the adjacent abdominal soft tissue. 


\section{Fig. 1. Surgical method of the four flaps technique}

Three points (the 3,6, and 9 o'clock abdominal flaps) were anchored to the deep dermis of the umbilicus and linea alba with a 4-0 absorbable suture. S, superior flap; $L$, lateral flap; I, inferior flap; $X_{1}$ anchoring point at the appropriate linea alba.

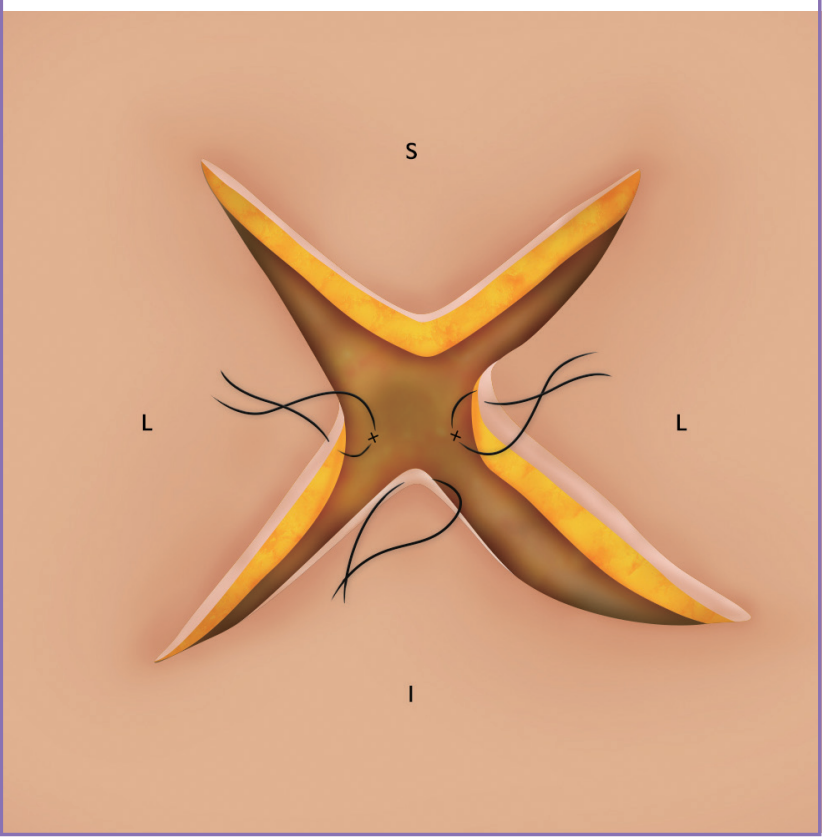

To determine the proper position of the neoumbilicus, we drew two axes on the abdomen as preliminary markings. The transverse axis was marked by connecting both anterior superior iliac spines of the iliac crests, and the vertical axis was drawn along the midline of the abdomen in a supine position. We designed an X-shaped incision line (Fig. 4). Each of the four triangular flaps had an angle of 90 degrees and the radius of each fan-shaped sector stretched $1 \mathrm{~cm}$ over the desired location of the neoumbilicus (Fig. 4). The original umbilicus of the patient in Fig. 3 consisted entirely of hypertrophic scar tissue, and the scarred umbilicus was completely resected before the reconstruction. After the en bloc excision, a defect was present with a diameter of 3 $\mathrm{cm}$, which led us to design an X-shaped incision line with limbs of $1.5 \mathrm{~cm}$ in length (Fig. 3). Under local anesthesia, a skin incision was made, resulting in four flaps in superior, inferior, and lateral positions. The four fan-shaped flaps were then completely detached from the underlying Scarpa's fascia, followed by a defatting procedure, although some subcutaneous fat was preserved at the base of the flap. The amount of fat preserved was dependent on the amount of abdominal fat present (Fig. 4). After meticulous hemostasis, the dermis of the three non-superior flaps was firmly anchored to the appropriate linea alba in the 3, 6, and 9 o'clock positions with 4-0 absorbable sutures (Vicryl, Ethicon, Somerville, New Jersey) to produce aesthetically acceptable folding (Fig. 5). In the case that a more longitudinal um-

\section{Fig. 2. A congenitally absent umbilicus}

The 40-year-old female patient showed a congenital absence of umbilical tissue and a soft tissue defect in the epigastric region.

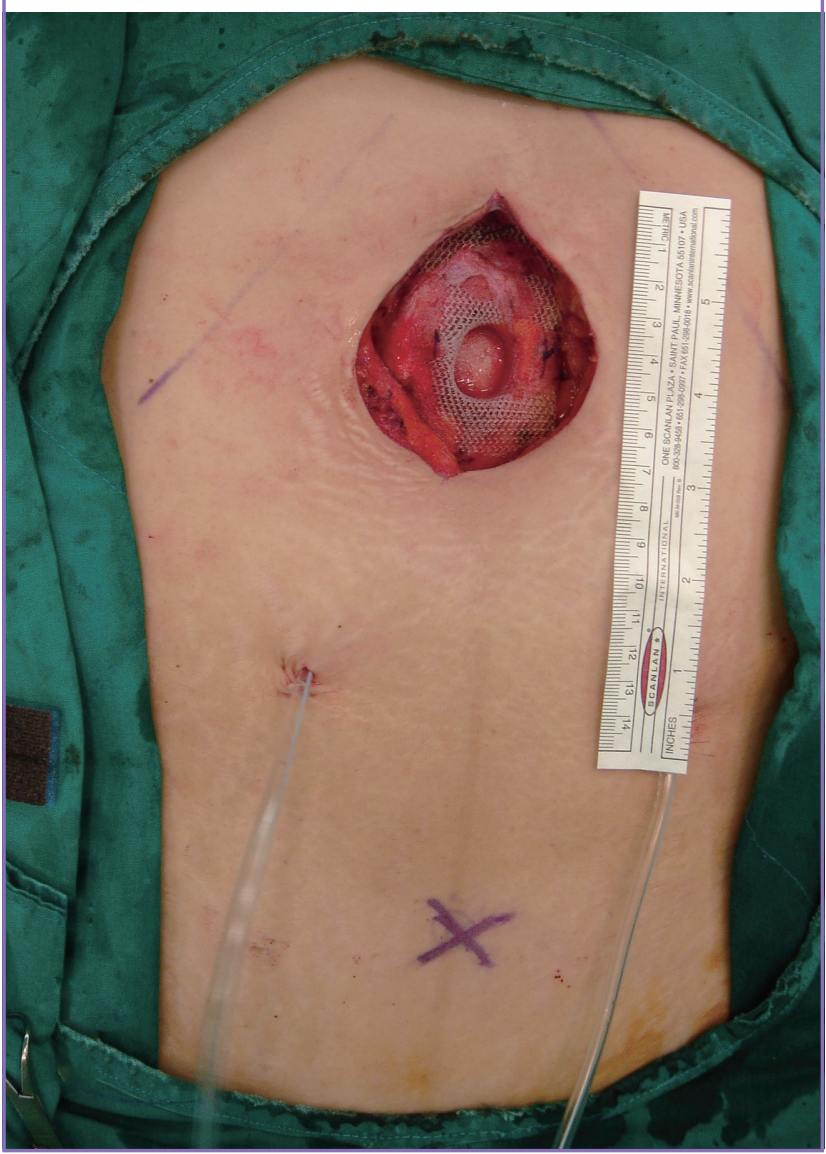

bilical depression is needed, the inferior flap could be anchored as caudally as possible. The remaining superior flap was not directly fixed to the underlying fascia. Instead, it was used to form the superior hooding; we performed a defatting procedure on the superior flap to a lesser extent than on other flaps, resulting in superior hooding (Fig. 4). The skin of each flap was then sutured with 5-0 non-absorbable nylon sutures (Ethicon, Somerville, New Jersey). The procedure was completed by packing a small amount of petrolatum gauze in order to ensure that the neoumbilicus remained satisfactorily hollow. Finally, the newly formed umbilicus was immobilized with a tie-over dressing to prevent movement postoperatively, which was maintained for one week. After two weeks, all of the stitches were removed and silicone gel (Scarease Gel, Pillar Surgical, La Jolla, CA, USA) was applied for scar management if necessary. There were no complications such as wound disruption, flap necrosis, hematoma, or infection. In each case, the neoumbilicus retained a sufficient depth during the follow-up period and both patients were satisfied with their post-operative results (Fig. 6). 
Fig. 3. A case of hypertrophic umbilicus

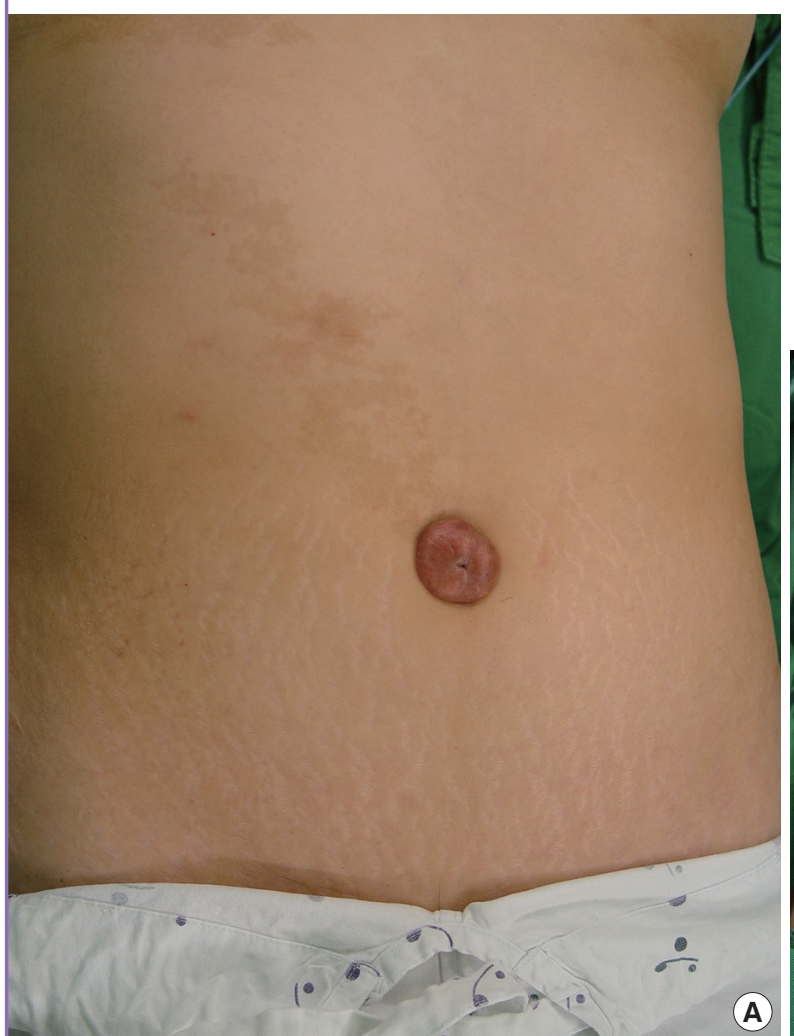

(A) Preoperative view of a 40-year-old female with hypertrophic umbilicus. (B) After en bloc excision of the hypertrophic scarred umbilicus, an X-shaped incision was designed, starting from the excised skin margin and consisting of four limbs, each $1.5 \mathrm{~cm}$ long.

Fig. 4. Operative procedures for the congenitally absent umbilicus

(A) The design of an X-shaped pattern consisting of four limbs with a length of $1 \mathrm{~cm}$. (B) The four flaps of the neoumbilicus were elevated and abdominal fat was removed.
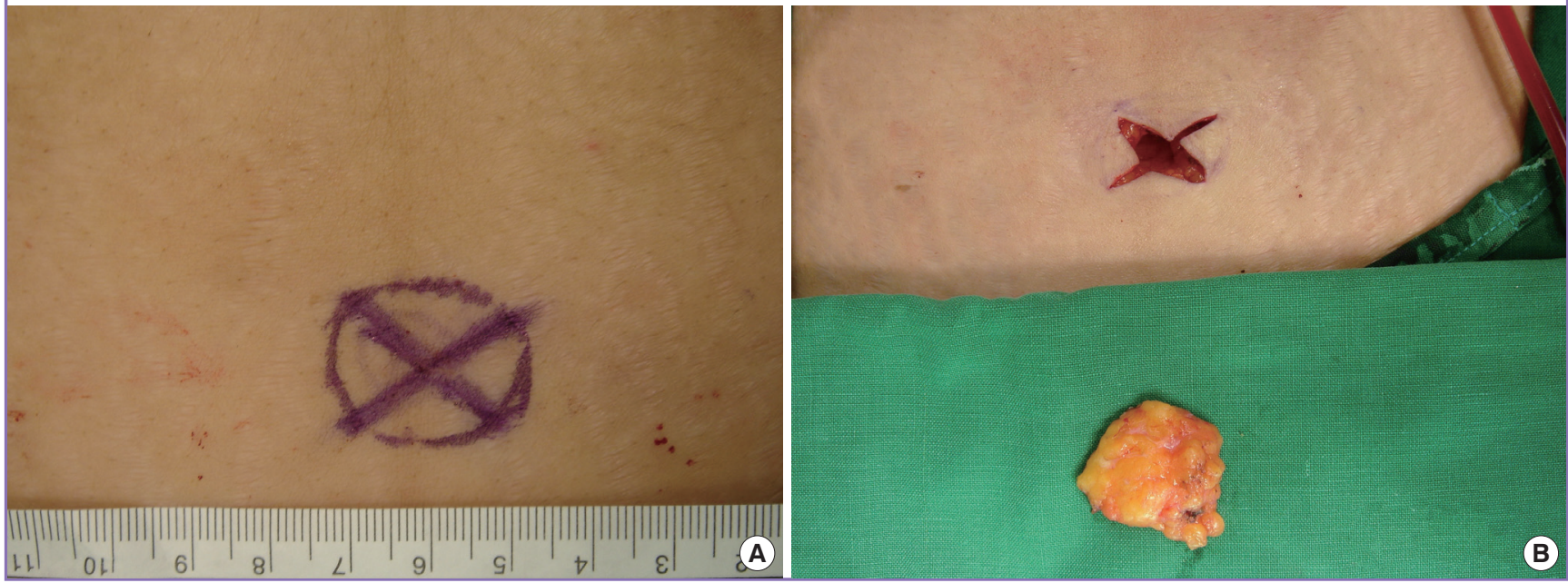

\section{DISCUSSION}

The umbilicus is located at the midpoint of the lower abdomen and is the major aesthetic unit of the abdominal wall. It is composed almost entirely of dermal and fibrotic tissue, along with a very small amount of subcutaneous fatty tissue, and is usually located approximately 9-12 $\mathrm{cm}$ above the superior margin of the mons pubis, slightly above a line connecting the anterior superior iliac spines [8]. The ideal umbilicus should have a natural contour, prominent depth, minimal additional scars, and proper superior hooding. Shinohara et al. [9] emphasized that an umbilicus with a natural appearance consists of a ring, a tubular 


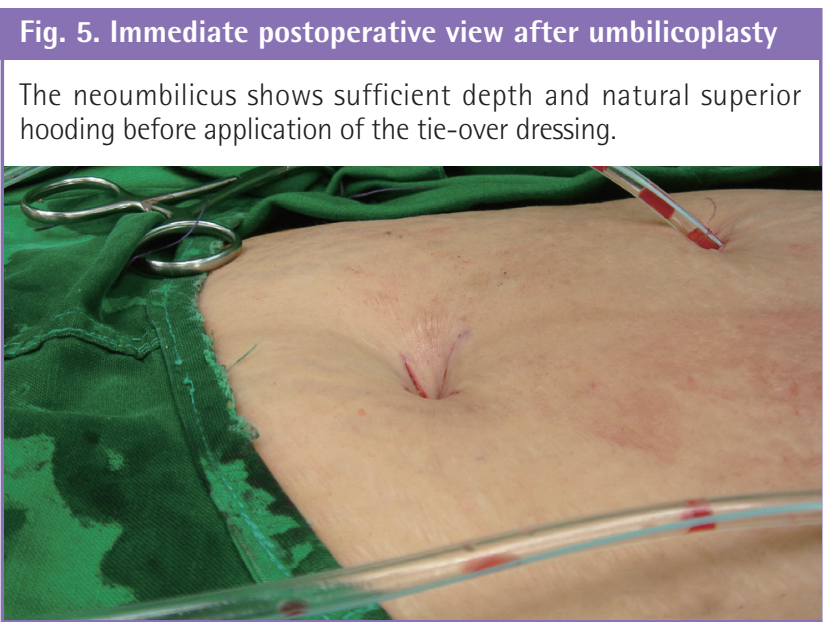

wall, a sulcus, and a bottom, without any excess skin that would interfere with the aesthetic aspect of the umbilicus. In addition, Lee et al. [10] suggested that an aesthetically optimal umbilicus must possess the following properties: a vertical ratio of 46:54 (with respect to the xiphoid process and the lower limit of the vulvar cleft), a midline horizontal position, and an oval shape with no hooding or superior hooding.

The absence of the umbilicus is an aesthetically and psychologically frustrating condition for patients, and sometimes poses challenges to surgeons [11]. It has recently become common for many people to want an aesthetically pleasing umbilicus because their umbilicus is visible in public when they wear clothes such as crop tops or bikinis. Since many patients have undergone abdominal reconstructive surgery, including procedures involving an inferior epigastric artery perforator flap, developing expertise in umbilical reconstruction has become obligatory for surgeons. However, few reports have addressed reconstructive neoumbilicoplasty after the en bloc excision of the umbilicus, as occurs in surgery treating a disfigured or hypertrophic umbilicus, umbilical hernia [5], neonatal omphalocele [12], or urachal cancer [13].

Several studies have suggested various surgical techniques for umbilical reconstruction, such as using diversely shaped local flaps, a suture method, and even cartilage grafts $[1-7,9,11,14]$. The local flap techniques, including an inverted C-V flap [3], yield good short-term results, but these techniques have not yet been used in the absence of remaining umbilical tissue. Miller and Balch [4] introduced the Iris technique for immediate umbilical reconstruction. This technique creates four flaps by making four curvilinear incisions followed by rotating the flaps after a wide resection of the umbilical tissue. However, this procedure leaves a conspicuous dog-ear scar around the umbilicus. Bartsich and Schwartz [1] reported a purse-string method for umbilical reconstruction after repairs of recurrent incisional hernia
Fig. 6. Postoperative view of the congenitally absent umbilicus

This figure shows the results eight years postoperatively, demonstrating the natural appearance of the neoumbilicus with sufficient depth, superior hooding, and minimal additional scarring.

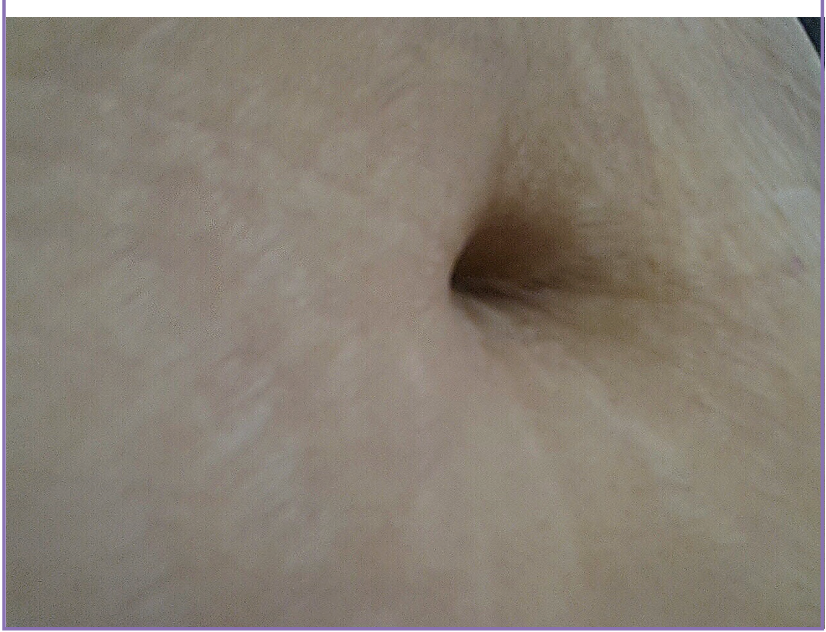

or abdominal surgical procedures such as urachal cyst repair. However, this technique requires thorough wound care for an extended period, as it leaves an open wound after the procedure. Pfulg et al. [2] introduced the use of a triangular flap for umbilical reconstruction after elliptical skin excision over the umbilicus in either a vertical or horizontal direction. It was simple and reproducible with few complications. However, it inevitably results in a long vertical or horizontal scar over the umbilicus to ensure that the neoumbilicus is adequately sized. The major disadvantage of the above techniques is that they use abdominal ground tissue from the abdominal wall. In practice, this is a challenging task for lighter patients and pediatric patients, especially in thin patients, because it is difficult to produce sufficient depth due to lower skin laxity. A neoumbilicus created using those methods may therefore develop a flat appearance over time.

In conclusion, the techniques described above are either unsatisfactory or too complex $[2,4,11]$. In contrast to those methods, we left only minimal scars embedded within the dimple. Our technique can also be performed on a flat abdominal wall without any remaining umbilical tissue or if there is a partial absence of abdominal skin after the removal of the umbilical tissue. It can also be applied as both a delayed and immediate reconstructive procedure with many advantages, including: (1) being simple and easy, (2) being appropriate for junior surgeons, (3) involving the manipulation of well-vascularized flaps, (4) yielding satisfactory superior hooding, and (5) resulting in a minimal external scar. Considering these advantages and the fact that it leads to aesthetically satisfactory results, this technique can be widely employed as a useful neoumbilicoplasty 
technique in patients suffering from an absent or disfigured umbilicus.

\section{REFERENCES}

1. Bartsich SA, Schwartz MH. Purse-string method for immediate umbilical reconstruction. Plast Reconstr Surg 2003; 112:1652-5.

2. Pfulg M, Van de Sijpe K, Blondeel P. A simple new technique for neo-umbilicoplasty. Br J Plast Surg 2005;58:688-91.

3. Hong YG, Cho JJ. Reconstruction of scarred umbilicus using an inverted c-v flap: a case report. J Korean Soc Plast Reconstr Surg 2007;34:653-5.

4. Miller MJ, Balch CM. "Iris" technique for immediate umbilical reconstruction. Plast Reconstr Surg 1993;92:754-6.

5. el-Dessouki NI, Shehata SM, Torki AM, et al. Double halfcone flap umbilicoplasty: a new technique for the proboscoid umbilical hernia in children. Hernia 2004;8:182-5.

6. Ricketts RR, Luck SR. Simultaneous umbilicoplasty and closure of small omphaloceles. Surg Gynecol Obstet 1983; 157:572-3.

7. Matsuo K, Kondoh S, Hirose T. A simple technique for re- construction of the umbilicus, using a conchal cartilage composite graft. Plast Reconstr Surg 1990;86:149-51.

8. Friedland JA, Maffi TR. MOC-PS(SM) CME article: abdominoplasty. Plast Reconstr Surg 2008;121:1-11.

9. Shinohara H, Matsuo K, Kikuchi N. Umbilical reconstruction with an inverted C-V flap. Plast Reconstr Surg 2000; 105 : 703-5.

10. Lee SL, DuBois JJ, Greenholz SK, et al. Advancement flap umbilicoplasty after abdominal wall closure: postoperative results compared with normal umbilical anatomy. J Pediatr Surg 2001;36:1168-70.

11. Kirianoff TG. Making a new umbilicus when none exists. Case report. Plast Reconstr Surg 1978;61:603-4.

12. Frigo E, Rettinger-Schimmerl S, Rokitansky AM. Umbilicoplasty in neonates with primary omphalocele closure. Pediatr Surg Int 1999; 15:523-4.

13. Park ES, Kim MS, Kim YB. Immediate umbilical reconstruction for urachal cancer. J Korean Soc Aesthetic Plast Surg 2003;9:59-62.

14. Marconi F. Reconstruction of the umbilicus: a simple technique. Plast Reconstr Surg 1995;95:1115-7. 